\title{
Hijack it, change it: how do plant viruses utilize the host secretory pathway for efficient viral replication and spread?
}

\author{
Camilo Patarroyo ${ }^{1}$, Jean-François Laliberté ${ }^{2}$ and Huanquan Zheng $^{1}$ * \\ ${ }^{1}$ Department of Biology, McGill University, Montreal, QC, Canada \\ ${ }_{2}$ INRS-Institut Armand-Frappier, Institut National de la Recherche Scientifique, Laval, QC, Canada
}

\author{
Edited by: \\ Peter Moffett, Université de \\ Sherbrooke, Canada \\ Reviewed by: \\ Keiko Yoshioka, University of Toronto, \\ Canada \\ Vicente Pallas, Instituto de Biología \\ Molecular y Celular de Plantas \\ Universidad Politécnica de Valencia- \\ Consejo Superior de Investigaciones \\ Científicas), Spain

\section{${ }^{*}$ Correspondence:} \\ Huanquan Zheng, Department of \\ Biology, Mc Gill University, 1205 \\ Doctor Penfield Avenue, Montreal, \\ QC, Canada H3A $1 B 1$. \\ e-mail: hugo.zheng@mcgill.ca
}

The secretory pathway of eukaryotic cells has an elaborated set of endomembrane compartments involved in the synthesis, modification, and sorting of proteins and lipids. The secretory pathway in plant cells shares many features with that in other eukaryotic cells but also has distinct characteristics important for fundamental cell and developmental processes and for proper immune responses. Recently, there has been evidence that the remodeling of this pathway, and often the formation of viral-induced organelles, play an important role in viral replication and spread. The modification of the host secretory pathway seems to be a common feature among most single-stranded positive ss(+)RNA and even some DNA viruses. In this review, we will present the recent advances in the understanding of the organization and dynamics of the plant secretory pathway and the molecular regulation of membrane trafficking in the pathway. We will also discuss how different plant viruses may interact with the host secretory pathway for their efficient replication and spread, with a focus on tobacco mosaic virus and turnip mosaic virus.

Keywords: ER, Golgi, endosomes, virus replication, virus transport

\section{INTRODUCTION}

Like any other eukaryotic cells, plant cells are characterized by an elaborated secretory pathway composed of a complex network of organelles including the endoplasmic reticulum (ER), the Golgi apparatus, the trans-Golgi network (TGN), various endosomes, and vacuoles (Hanton et al., 2005; Bassham et al., 2008). This pathway is involved in the synthesis, modification and transport of proteins, lipids and polysaccharides (Bassham et al., 2008). Proteins made in the ER can be transported, via Golgi, toward the plasma membrane; on the other hand, proteins outside of the cells can also be internalized, via endocytosis. The proper organization and the dynamics of the secretory pathway are vital for normal cell development and physiology (Hanton et al., 2005).

In animal cells, it is known that efficient replication of many viruses involves a modification of the secretory pathway in host cells that generates a membrane structure designated as virosome, virus inclusion, virus factory, or viroplasm (for review, see Novoa etal., 2005; Netherton etal., 2007; Miller and Krijnse-Locker, 2008). Most animal viruses are membrane enveloped and it is known that the modification of the secretory pathway in host cells is also crucial for viral assembly (Netherton et al., 2007), intracellular and intercellular movement of viral complexes (Brandenburg and Zhuang, 2007), and inhibition of cellular secretion to reduce host immune response (Netherton et al., 2007). Different animal viruses modify the secretory pathway in many different ways for efficient viral replication, assembly, and spread along the secretory pathway (Netherton et al., 2007). For example, picornaviruses modify the early secretory pathway for RNA replication and inhibition of cellular secretion by interfering with the function of Arf1 and its guanine nucleotide exchange factor GBF1 in the formation of COPI vesicles (Hsu et al., 2010), while Norwalk virus inhibits cellular secretion through an interaction with COPII vesicles (Sharp et al., 2010). It is interesting that, although much less is known about how plant viruses modify the plant secretory pathway, recent data indicate that many plant viruses also utilize the plant secretory pathway for efficient replication and probably for cell-to-cell spread (Laliberte and Sanfacon, 2010; Schoelz et al., 2011; Verchot, 2011).

In this review, we will first highlight the recent progress in the organization and dynamics of the plant secretory pathway, we will then present the recent findings on how plant viruses may utilize the host secretory pathway for replication and intracellular and intercellular transport, with a focus on the recent advances on how the secretory pathway may be utilized by tobacco mosaic virus (TMV) and turnip mosaic virus (TuMV) for successful virus infection.

\section{THE ORGANIZATION AND DYNAMICS OF THE ER-GOLGI INTERFACE IN PLANT CELLS}

The ER is an extensive membrane network that extends throughout the cytoplasm. The ER is the largest membranous structure in the eukaryotic cell; it is the site where proteins and lipids are synthesized and modified. The Golgi apparatus, with cis-Golgi facing the ER and trans-Golgi away from the ER, is the central station in the secretory pathway. In Golgi, proteins and lipids received from the ER will be further modified and sorted to the proper destination in the secretory pathway. Despite the fact that in animal cells the ER is mainly aligned with microtubules (Du et al., 2004) and in plant cells with microfilaments (Boevink et al., 1998), the morphology of the ER in plants is quite similar to that in animal cells. The ER in both mature animal and plant cells exhibits a labyrinth-like morphology composed of membranous tubules and cisternae (Hu et al., 2009; Orso et al., 2009; Chen et al., 2011, 2012). In addition, the mechanisms that mediate the tubulation of 
the ER (Voeltz et al., 2006; Sparkes et al., 2010) as well as the generation of interconnected ER network also appear to be conserved across different eukaryotic cells (Hu et al., 2009; Orso et al., 2009; Chen etal., 2011; Zheng and Chen, 2011). On the other hand, the organization of the Golgi apparatus is quite different between mammalian and plant cells. In mammalian cells, Golgi stacks form a single large perinuclear ribbon at the microtubule organization center peripheral to the nucleus (Ladinsky et al., 1999); in plant cells, the Golgi apparatus is present in the form of numerous individual cisternal stacks that move rapidly along the actin/ER cable in the cytoplasm (Boevink et al., 1998). As such, the morphology of the protein transport in the ER-Golgi interface appears very different between mammalian and plant cells.

Transport of proteins from the ER to the Golgi apparatus in both animal and plant cells starts from a transitional ER domain called ER export site (ERES). In mammalian cells, the ERESs are relatively stationary (Hammond and Glick, 2000), which are linked with the cis-Golgi by ERGIC, an ER-Golgi intermediate compartment made by the fusion of COPII vesicles formed at the ERESs (will be discussed below). In plant cells, individual ERESs are believed to be motile and tightly associated with Golgi stacks (daSilva et al., 2004). The ERES works together with individual Golgi stacks as a single functional unit (daSilva et al., 2004); no intermediate ERGIC has been revealed in plant cells. Yet it appears that the molecular mechanisms underlying protein transport in the ER-Golgi interface are very conserved between animal and plant kingdoms (Lee and Miller, 2007; Hsu and Yang, 2009; Hwang and Robinson, 2009; Marti et al., 2010; Chen et al., 2012).

In animal cells, transport from the ER to the Golgi apparatus is mediated by COPII vesicles (Lee and Miller, 2007). The proteins required for the formation of COPII vesicles from the ER include small Sar1 GTPase, membrane anchored guanine exchange factor (GEF) Sec12, Sec23/Sec24 heterodimers for cargo selection and initiation of ER membrane curving (Bickford et al., 2004), and Sec13/Sec31 for the final formation of cage-like spherical vesicle (Lee and Miller, 2007). The fusion of COPII vesicles to generate ERGIC, which will further mature into cis-Golgi cisternae, requires small Rab1 GTPase and ER-localized SNARE (soluble $N$ ethylmaleimide-sensitive-factor adaptor-protein receptors) proteins (Bonifacino and Glick, 2004). In plant cells, despite the morphological difference in the ER-Golgi interface, all these key proteins exist (Bassham et al., 2008; Marti et al., 2010). It has been experimentally demonstrated that AtSEC24a as well as Rab-D proteins, homologs of Rab1, are required for ER-to-Golgi transport in plant cells (Batoko et al., 2000; Faso et al., 2009; Qi and Zheng, 2011). In mammalian cells, the retrograde Golgi transport, essential for recycling of proteins and lipids back to the ER in order to maintain equilibrium with the COPII-dependent transport, occurs mainly through COPI vesicles (Hsu and Yang, 2009). The COPI coatomer is composed by two protein complexes, $\mathrm{F}$ - and BCOP. The formation of COPI vesicles in Golgi cisternae requires small Arf1 GTPase, whose activity is regulated by Sec7-domain Arf1-GEFs (Hsu and Yang, 2009). Brefeldin A (BFA), a fungal metabolite, has been found to be a specific inhibitor that interferes with the interaction between Sec7-domain Arf-GEF and Arf proteins (Nebenfuhr et al., 2002). In Arabidopsis thaliana, electron tomography analysis have shown the existence of two types of
COPI coated vesicles, the COPIa vesicles derived from cis-Golgi and the COPIb vesicles derived from the trans-Golgi (Hwang and Robinson, 2009).

\section{THE ORGANIZATION AND DYNAMICS OF POST-GOLGI TRAFFIC NETWORK IN PLANT CELLS}

Proteins and lipids, after being transported and modified in the Golgi apparatus, are further transported to either the plasma membrane, or to the lysosome/vacuole (in plant cells). In mammalian cells, cargo molecules modified in the Golgi apparatus are usually delivered to the TGN where they are sorted into either secretory vesicles that move to the plasma membrane, or clathrincoated vesicles to deliver cargoes to the endosomes and then the lysosome (Traub and Kornfeld, 1997). This anterograde protein transport to the plasma membrane is usually balanced by various clathrin-coated vesicle mediated endocytic pathways (Gruenberg and Maxfield, 1995; Clague and Urbe, 2001). After being formed in the plasma membrane, clathrin-coated endocytic vesicles are first delivered to the early endosome (EE), a compartment that can be marked by Rab5, a small GTPase required for the fusion of clathrin-coated vesicles to the EE (Gorvel etal., 1991; Rink et al., 2005). At the EE, proteins can be either recycled back to the plasma membrane via recycling endosomes (RE; Grant and Donaldson, 2009), or further sorted to the late endosomes (LE) and the lysosome. It is thought that the protein recycling at the EE via the RE is mediated by Rab11 (Grant and Donaldson, 2009). The LE is believed to mature by the fusion of different EEs mediated by small Rab7 GTPase and LE-specific SNARE proteins (Bonifacino and Glick, 2004; Rink et al., 2005). At the LE, ESCRT (the endosomal sorting complexes required for transport) at the surface can selectively pick ubiquitinated membrane proteins to be directed to the lysosomes for further degradation (Katzmann et al., 2001).

In plant cells, initially the TGN was thought to be absent, but recent evidence suggests that the TGN may be a motile organelle derived from the Golgi apparatus (Dettmer et al., 2006; Kang et al., 2011; Qi et al., 2011). It has been demonstrated that the plant TGN could act as a sorting station and simultaneously release two types of vesicles: secretory vesicles heading to the plasma membrane and the cell wall (Preuss et al., 2006; Szumlanski and Nielsen, 2009; Qi et al., 2011), and clathrin-coated vesicles mediating transport to vacuoles (Kang etal., 2011). The organization of the dynamics of the post-Golgi traffic network in plant cells is, however, not well studied. It appears that protein transport in the plant postGolgi traffic network is quite distinct from that in mammalian cells. There are several lines of evidence suggesting that the TGN in plants also serves as an EE (Dettmer et al., 2006; Qi et al., 2011). At the TGN/EE, proteins can be either recycled back to the plasma membrane (Ueda et al., 2001), or further transported to multivesicular bodies (MVB) or prevacuolar compartments (PVC), a compartment equivalent to the LE in animal cells (Spitzer et al., 2009). In plant cells, no RE has been identified. Different from mammalian cells, in plant cells the TGN/EE is highlighted by the presence of different Rab-A proteins (Szumlanski and Nielsen, 2009; Kang et al., 2011; Qi et al., 2011), homologs of the animal Rab11 protein; the MVB/PVC is marked by Rab-F proteins (Ueda et al., 2001), homologs of the Rab5 in animal cells. MVB/PVC 
bearing ESCRT that can selectively pick ubiquitinated membrane proteins has also been reported (Spitzer et al., 2009).

\section{THE ROLE OF THE HOST SECRETORY PATHWAY IN PLANT VIRAL REPLICATION}

Viruses are obligate intracellular parasites that depend on cellular materials for their replication and spread. As mentioned in the introduction, replication and assembly of many membrane enveloped animal viruses occur in an intracellular compartment termed virus factory, viral inclusion, viroplasm, or virosome whose generation requires the modification of the host secretory pathway (Novoa et al., 2005; Netherton et al., 2007; Miller and Krijnse-Locker, 2008). Despite the fact that most plant viruses are not membrane enveloped, recent research revealed that plant viruses also utilize the host secretory pathway for viral replication (Laliberte and Sanfacon, 2010; Verchot, 2011).

Studies with TMV, an extensively studied member of tobamoviruses, showed that TMV infection causes severe modifications of the ER, converting it into large irregular aggregates early in infection (Reichel and Beachy, 1998). This ER-derived structure contains virus particles, the virus-encoded 183 and $126 \mathrm{kDa}$ replicases, movement protein (MP), vRNA, ribosomes, and host translation elongation factor EF1- $\alpha$ (Beachy and Zaitlin, 1975; Heinlein et al., 1998; Reichel and Beachy, 1998; Mas and Beachy, 1999; Figueira et al., 2002). Therefore it is believed that this ER-derived structure is the site for TMV replication, which is often termed as viral replication complex (VRC; Heinlein et al., 1998). However, how exactly these TMV replication complexes are formed is not yet clear. The MP and the replicase components 126 and $183-\mathrm{kDa}$ proteins are associated with the ER (Reichel and Beachy, 1998; Hagiwara et al., 2003). The MP, when expressed ectopically, can induce modifications in the ER (Reichel and Beachy, 1998) and the $126-\mathrm{kDa}$ replicase, when expressed ectopically, can also induce the formation of cytoplasmic inclusion bodies (Ding et al., 2004). Therefore it is possible that these viral proteins are involved in the formation of TMV replication complexes. Furthermore, the TMV replication complexes are found to be associated with the host transmembrane proteins TOM1 (Yamanaka et al., 2000; Hagiwara et al., 2003) and ARL8, a small GTP-binding protein (Nishikiori et al., 2011). Both proteins are required for efficient TMV RNA replication (Nishikiori et al., 2011). This suggests that host proteins also play important roles in the formation of TMV replication complexes in the secretory pathway. It will be interesting to study how exactly these viral and host proteins are involved in the process.

In the case of potyviruses, TuMV infection induces the formation of small vesicular compartments that move rapidly along the microfilaments and the ER (Beauchemin et al., 2007; Cotton et al., 2009; Grangeon et al., 2012). The polyprotein $6 \mathrm{~K}_{2}$-VPg-Pro, through its hydrophobic $6 \mathrm{~K}_{2}$ domain, is responsible for the formation of these motile structures (Beauchemin et al., 2007). The presence of viral dsRNA, an obligate intermediate in the replication of the ssRNA viruses, as well as the host eukaryotic initiation factor iso4E ( $\mathrm{eIF}$ (iso)4E), poly-A binding protein, heat shock cognate 70-3 protein (Hsc70-3), and the eukaryotic elongation factor $1 \mathrm{~A}$ (eEF1A; Beauchemin and Laliberte, 2007; Beauchemin et al., 2007; Dufresne et al., 2008; Thivierge et al., 2008; Cotton et al., 2009) in the $6 \mathrm{~K}_{2}$-induced vesicles suggests that the viral genome replication takes place in these structures (Cotton et al., 2009). Interestingly, in cells infected by the potyvirus tobacco etch virus (TEV), the motile $6 \mathrm{~K}_{2}$ vesicular compartments are ER-derived and are associated with ERESs. The formation of the structure is COPII- and COPI-dependent (Wei and Wang, 2008). The motile $6 \mathrm{~K}_{2}$ vesicles in TuMV-infected cells are associated but not perfectly with COPII vesicles as well as Golgi stacks (Grangeon et al., 2012). BFA can enhance the association of $6 \mathrm{~K}_{2}$ vesicles with COPII and Golgi stacks (Grangeon et al., 2012). The interpretation of these facts is that the formation as well as the transport (will be discussed further) of the motile $6 \mathrm{~K}_{2}$ structures requires an interaction between $6 \mathrm{~K}_{2}$ and host proteins involved in the formation and transport of COPII and COPI vesicles.

Recently, we reported that TuMV induces the formation of a perinuclear globular structure with an amalgamation of viral $6 \mathrm{~K}_{2}$, ER, Golgi, and COPII membranes as well as the presence of chloroplasts within the structure (Grangeon et al., 2012). This globular structure maintains a dynamic connection with the cortical ER and the Golgi apparatus; additionally the motile $6 \mathrm{~K}_{2}$ vesicles may originate from the globular structure (Grangeon et al., 2012). We hypothesize that this globular structure could provide an extended platform for viral replication and protein synthesis (Grangeon et al., 2012). Indeed, the formation of large perinuclear ER-derived structures has also been observed in several other plant viruses. For example, cowpea mosaic virus (CPMV) induces the proliferation of ER membranes (de Zoeten et al., 1974; Carette et al., 2000, 2002a) that are often next to the nuclei. By the incorporation of $\mathrm{H}^{3}$-uridine in the infected leaves, newly synthesized viral RNA can be found in the perinuclear membranous structures induced by CPMV (de Zoeten et al., 1974). Grapevine fanleaf virus (GFLV) induces the formation of a punctate-spongy perinuclear structure composed of reorganized ER membranes and active synthesis of RNA is found in these ER-derived structures (Ritzenthaler et al., 2002). Potato virus X (PVX) also forms an ER-contained perinuclear structure called X-body (Tilsner et al., 2012) where the viral replicase and TGB1 are located.

The exact mechanism by which these globular structures are formed remains unclear. It is known in plant cells that disruption of the formation of COPII vesicles also induces the formation of perinuclear clusters of the ER and Golgi membranes (Faso et al., 2009). The $60 \mathrm{~K}$ helicase of CPMV interacts with the ER localized vSNARE-like protein VAP27 in the proliferation of ER membranes (Carette et al., 2002b), suggesting that an interaction of viral and host ER proteins may be required in the process. It is interesting, however, in TuMV infected cells, the BFA treatment does not inhibit the formation of the perinuclear globular structure nor viral replication (Grangeon et al., 2012). Although the perinuclear structure in TuMV-infected cells contains the ER and Golgi membranes, it seems that the formation of the structure does not require a functional early secretory pathway. Similarly, BFA seems not affect replication of melon necrotic spot virus but it may have a negative impact on viral cell-to-cell movement (Genoves et al., 2010). On the other hand, in GFLV-infected cells, although it is not known if the BFA treatment inhibits the formation of the perinuclear structure, such treatment results in a significant reduction of the replication efficiency of the virus (Ritzenthaler 
et al., 2002). It is highly likely that different viruses use different strategies to generate membranous structures for their efficient replication.

In animal cells, the generation of the virus factory containing the membranous structures of the host secretory pathway is also thought to be a mechanism for viruses to hide from host cell anti-viral defenses (Novoa et al., 2005; Miller and KrijnseLocker, 2008). With regards to this, it is interesting to note that in $N$. benthamiana cells expressing a dominant-negative Vps23, Vps24, Snf7p, and Vps4p, components of ESCRT complexes, the replication of the tomato bushy stunt virus (TBSV), a member of tombusvirus, is impaired (Barajas et al., 2009). In addition, in yeast $v p s 23 \Delta$ or $v p s 24 \Delta$ cells, the activity of the viral replicase $\mathrm{p} 92$ is reduced and the $(-)$ stranded viral RNA is more accessible to ribonucleases (Barajas et al., 2009). How the absence of ESCRT complexes in host cells could lead to a reduced activity of p92 and less stable viral RNA remains unknown. As mentioned previously, the ESCRT complexes are required for the recognition and sorting of ubiquitin-modified cargo proteins into MVBs and eventually to the vacuole (Katzmann et al., 2001; Spitzer et al., 2009). It is possible that ESCRT complexes are recruited into the viral replicase complex at some stages to help the virus to evade the recognition by host defense proteins and/or to avoid the degradation by the gene silencing machinery. Indeed, the p33 replication protein of TBSV interacts with Vps23p (Barajas et al., 2009), suggesting that the viral protein may be involved in the recruitment of ESCRT into the virus factory, or regulate the activity of ESCRT in the factory.

Although most plant viruses are not membrane enveloped, it is interesting to note that there is a small group of plant viruses such as the tomato spotted wilt virus (TSWV) that are membrane enveloped (Mumford et al., 1996). TSWV is a plantinfecting counterpart of the animal infecting viruses within the arthropod-borne Bunyaviridae. It is known that bunyaviruses that infect animal cells are replicated in tubular viral factories that are built around Golgi stacks but are connected to both mitochondria and the rough ER (Walter and Barr, 2011). After the final virion assembly in the lumen of swollen Golgi stacks, viruses bud into secretory vesicles in which they migrate toward the plasma membrane and are then secreted (Walter and Barr, 2011). However, it is not clear if this is also the case for TSWV. TSWV is replicated in infected plant cells in association with a membrane modification of the ER and Golgi (Ribeiro et al., 2008). The viral particles are assembled within the infected plant cells when ribonucleoprotein complexes (RNPs) are enwrapped by Golgi cisternae. The Golgi membrane wrapped RNPs then fuse with each other and with ER membranes, producing a singly enveloped vesicle where virus particles are retained until uptake by its insect vector (Kitajima et al., 1992; Kikkert et al., 1999). It was recently shown that the TSWV glycoproteins Gn and Gc induce the formation of ER- and Golgiderived membranous structures upon ectopic expression in plant protoplasts (Ribeiro et al., 2008). The Gn glycoprotein localizes to both the ER and Golgi and is able to redirect the ER-arrested glycoprotein Gc to Golgi in a COPII-dependent manner upon co-expression (Ribeiro et al., 2009). In addition, both Gn and Gc interact with the TSWV nucleocapsid protein (N) and RNPs in ER and Golgi (Ribeiro et al., 2009). Therefore it seems that viral
Gn and Gc glycoproteins, transported within the ER and Golgi, play an important role in the generation of the hybrid ER-Golgi structure where the virus is replicated and assembled. As one of the few plant viruses that are enveloped by lipid membranes, it will be interesting to examine how TSWV migrates, once assembled in the cytoplasmatic vesicles, to the neighboring cells. Likely, TSWV moves cell-to-cell differently from its animal counterpart, because the viral NSm protein, which was shown to restore the cell-to-cell and long distance movement of a movement-defective TMV clone (Lewandowski and Adkins, 2005), forms tubules in association with plasmodesmata (PD) upon TSWV infection of N. rustica (Storms et al., 1995).

\section{ROLE OF THE HOST SECRETORY PATHWAY IN INTRA- AND INTER-CELLULAR TRANSPORT OF VIRUSES}

As described above, many plant viruses replicate in infected cells in association with membranous structures derived from the host secretory pathway (Laliberte and Sanfacon, 2010; Verchot, 2011). Consequently, virions or viral nucleic acid-protein complexes must travel from the site where they are replicated to the neighboring cells to start the replication cycle again in order to cause a systemic infection. It has been generally accepted that plant viruses move from an infected cell to its neighboring cells through PD (Schoelz et al., 2011; Ueki and Citovsky, 2011). However, the virions and even the naked genome of plant viruses are too large to fit through unaltered PD. To facilitate the spread of viral particles or replication complexes, most plant viruses encode at least one dedicated protein termed movement protein (Lucas, 2006) to modify the structure of PD (Lucas, 2006; Schoelz et al., 2011; Ueki and Citovsky, 2011).

How could viral particles or replication complexes generated in the virus factory move to PD? Studies of various plant viruses over the past decades indicated that host cytoskeletal elements provide viruses the means to move from their sites of replication to PD from where they travel to the neighboring cells (Schoelz et al., 2011; Ueki and Citovsky, 2011). However, recent studies of some plant viruses indicate that the host secretory pathway and protein transport machineries may also be hijacked by viruses for their efficient transport to PD and cell-to-cell movement. In TMV, the MP and the $126 \mathrm{kDa}$ replicase proteins are required for its efficient movement (Reichel and Beachy, 1998; Hirashima and Watanabe, 2001). Both proteins as well as TMV replication complexes are found to be associated with the ER (Heinlein et al., 1998; Yamanaka et al., 2000; Hagiwara et al., 2003). However, it is interesting that lower concentrations of BFA $(10 \mu \mathrm{g} / \mathrm{ml})$ or overexpression of dominant negative Sar1 does not inhibit intercellular transport of neither the MP of TMV nor the TMV replication complexes to PD (Tagami and Watanabe, 2007), while a higher concentration of BFA (>50 $\mu \mathrm{g} / \mathrm{ml})$ did inhibit the movement of the TMV MP to PD (Heinlein et al., 1998; Wright et al., 2007). The higher concentration of BFA is known to disrupt interconnected ER network (Zheng et al., 2004). Thus the interpretation of the different effect of different BFA concentrations could be that the transport of TMV to PD requires a networked ER but does not require the COPII-/COPI-dependent molecular machinery. Considering the fact that ER tubules traverse through PD to the neighboring cells, it is possible that ER tubules are used directly 
by TMV as a transport conduit for both intra- and inter-cellular transport.

In the case of the members of the genus Potyviruses, it appears that intracellular transport of virus replication complexes from the site where they replicate to $\mathrm{PD}$ requires the classic COPII/COPI vesicle trafficking machinery in the secretory pathway. As described in the previous section, in both TuMV- and TEV-infected cells, motile $6 \mathrm{~K}_{2}$ vesicular structures are identified as a site where viral genome can be replicated (Beauchemin et al., 2007; Cotton et al., 2009; Grangeon et al., 2012). Yet the formation of these motile $6 \mathrm{~K}_{2}$ vesicles is COPII- and COPI-dependent (Wei and Wang, 2008). They are generated from ERESs, move to Golgi stacks, and are transported along the actin and the ER to the cell periphery (Beauchemin et al., 2007; Wei and Wang, 2008; Cotton etal., 2009; Grangeon et al., 2012). Furthermore, the movement of $6 \mathrm{~K}_{2}$ and the cell-to-cell movement of TuMV can be inhibited by BFA at $10 \mu \mathrm{M}$ (Grangeon et al., 2012). For potyviruses, the proteins $\mathrm{VPg}, \mathrm{CI}, \mathrm{CP}$, and $\mathrm{P} 3 \mathrm{~N}-\mathrm{PIPO}$ have been implicated in inter-cellular movement of viruses through $\mathrm{PD}$ (Nicolas et al., 1997; Rojas et al., 1997; Carrington et al., 1998; Wei and Wang, 2008; Wei et al., 2010). It is interesting that recently, Wei et al. (2010) demonstrated that the trafficking of P3N-PIPO and CI to PD requires a COPII-/COPI-dependent protein transport machinery, but not the actomyosin system (Wei et al., 2010) as the localization of P3N-PIPO and CI to PD is abolished in infected cells treated with BFA, or in cells expressing a mutant version of Sar1 [Sar1(H74L)], but not by actin disrupting agents. It seems that the transport of the potyvirus replication complex to the cell periphery requires the classic COPII/COPI vesicle trafficking machinery in the secretory pathway and the actomyosin system, but the transport of the viral proteins to PD for the subsequent modification of PD only requires the classic COPII/COPI vesicle trafficking machinery in the secretory pathway. However, NSvc4, the putative MP of rice strip virus (RSV) and MP17, the MP of potato leaf roll virus (PLRV), when expressed alone, are targeted to PD in COPII- and actin-dependent manners (Vogel et al., 2007; Yuan et al., 2011). Similarly, the MP protein p7B of melon necrotic spot virus is found to localize to the ER, Golgi, and PD when ectopically expressed (Genoves et al., 2010). Genoves et al. (2010) show that both BFA and latrunculin B inhibit the transport of p7B to Golgi and PD at the level the ER. However, it is generally accepted that in plant cells the actomyosin system is not required for protein transport from the ER to Golgi (Brandizzi et al., 2002).

As mentioned previously, in plant virus infected cells, PD has to be modified, either with an increase in SEL or formation of tubules (reviewed by Schoelz et al., 2011), to allow viral particles or replication complexes to spread. Recent data suggested that, in addition to movement-related proteins encoded by various viruses, some host proteins, for example, PDLP1, a type-I membrane protein located to $\mathrm{PD}$, appear to be required for successful modification of $\mathrm{PD}$ (Huang et al., 2001; Thomas et al., 2008; Amari et al., 2010). When Arabidopsis plants expressing mutant versions of $P D L P 1, P D L P 2$, and $P D L P 3$ are inoculated with cauliflower mosaic virus (CaMV), a Caulimovirus that induces tubules through which CaMV virions move, significantly fewer plants present systemic infections (Amari et al., 2010). The replication of CaMV in the protoplasts carrying the triple mutation is, however, comparable to the replication in wild type Arabidopsis (Amari et al., 2010). This suggests that the PDLP1 protein plays a vital role in the movement of the virus, possibly in the modification of PD for the formation of tubules. It is interesting that the targeting of PDLP1 to PD is COPII-dependent (Thomas et al., 2008). However, in CaMVinfected cells, it appears that targeting of the MP of the virus to the cell periphery is not abolished by the treatment of BFA (Huang etal., 2000). It seems that transport of viral proteins of CaMV and host proteins to PD relies on different transport routes.

In addition to the above mentioned roles that the host secretory pathway may play in the transport of virus replication complexes and viral and host proteins required for efficient spread of viruses, it is worth noting that the TGB2 protein of potato mot top virus (PMTV), which is located to both the ER and PD and is probably involved in the modification of PD for intercellular virus movement (Cowan et al., 2002), can also be found in vesicles marked by FM4-64 (Haupt et al., 2005), a fluorescent dye that is widely used to track endocytosis in plant cells (Qi et al., 2011). TGB2 interacts with a J-domain chaperone essential for the endocytic protein transport and is also co-localized with Ara7, a Rab-F protein localized to the PVC/MVB (Haupt et al., 2005). In addition to this, the MP of TMV and a DNA virus, cabbage leaf curl virus (CaLCuV), physically interact with the clathrin-associated SNARE-interacting protein synaptotagmin (Lewis and Lazarowitz, 2010). Synaptotagmin is a $\mathrm{Ca}^{2+}$ sensor and down-regulation of this protein inhibits endocytosis and intercellular spread of TMV and CaLCuV (Lewis and Lazarowitz, 2010). However, the exact role that this endocytic protein transport pathway may play in the virus multiplication is yet to be determined. Endocytosis is known to be a mechanism for cells to regulate the localization and/or function of some plasma membrane localized proteins (Gruenberg and Maxfield, 1995), perhaps the accumulation and function of proteins required for modification of PD for efficient virus spread needs to be regulated by endocytosis.

\section{CONCLUDING REMARKS}

Plant cells have a sophisticated secretory pathway that is vital for their growth and survival in response for prevailing environmental conditions. In recent years, there has been an explosion of information regarding the organization of the plant secretory pathway and the molecular mechanisms that regulate protein transport in the plant secretory pathway. For successful infection of host plant cells, it seems that plant viruses have developed different yet highly host-adapted strategies to take advantage of the molecular machineries provided by the host secretory pathway to replicate and move from infected to uninfected cells. However, how plant viruses interplay with their host secretory pathway is not well understood. It is highly likely that different plant viruses interact differently with the host secretory pathway for efficient virus multiplication. To unravel the exact mechanisms by which different viruses are replicated, and transported to and through PD to neighboring cells, we need to identify more of the host factors required for the multiplication of different viruses. We need to understand how these host factors interact with different viral factors and to figure out the functional significance of these interactions in viral 
infection. We believe that a further understanding of these issues is important for those who wish to develop plant cultivars that resist to virus infection. On the other hand, such research could also serve as a way to unlock the secrets of the host secretory pathway in plant cells.

\section{REFERENCES}

Amari, K., Boutant, E., Hofmann, C., Schmitt-Keichinger, C., FernandezCalvino, L., Didier, P., et al. (2010). A family of plasmodesmal proteins with receptor-like properties for plant viral movement proteins. PLoS Pathog 6:e1001119. doi: 10.1371/journal.ppat.1001119

Barajas, D., Jiang, Y., and Nagy, P. D. (2009). A unique role for the host ESCRT proteins in replication of tomato bushy stunt virus. PLoS Pathog 5:e1000705. doi: 10.1371/journal.ppat.1000705

Bassham, D. C., Brandizzi, F., Otegui, M. S., and Sanderfoot, A. A. (2008). The secretory system of Arabidopsis. Arabidopsis Book 6:e0116. doi: 10.1199/tab.0116

Batoko, H., Zheng, H. Q., Hawes, C., and Moore, I. (2000). A Rab1 GTPase is required for transport between the endoplasmic reticulum and Golgi apparatus and for normal Golgi movement in plants. Plant Cell $12,2201-2218$

Beachy, R. N., and Zaitlin, M. (1975). Replication of tobacco mosaic virus: VI. Replicative intermediate and TMV-RNA-related RNAs associated with polyribosomes. Virology 63, 84-97.

Beauchemin, C., Boutet, N., and Laliberte, J. F. (2007). Visualization of the interaction between the precursors of VPg, the viral protein linked to the genome of turnip mosaic virus, and the translation eukaryotic initiation factor iso $4 \mathrm{E}$ in planta. J. Virol. 81, 775-782.

Beauchemin, C., and Laliberte, J. F. (2007). The poly(A) binding protein is internalized in virus-induced vesicles or redistributed to the nucleolus during turnip mosaic virus infection. J. Virol. 81, 10905-10913.

Bickford, L. C., Mossessova, E., and Goldberg, J. (2004). A structural view of the COPII vesicle coat. Curr. Opin. Struct. Biol. 14, 147-153.

Boevink, P., Oparka, K., Santa Cruz, S., Martin, B., Betteridge, A., and Hawes, C. (1998). Stacks on tracks: the plant Golgi apparatus traffics on an actin/ER network. Plant J. 15, 441-447.

Bonifacino, J. S., and Glick, B. S. (2004). The mechanisms of vesicle budding and fusion. Cell 116, 153-166.
Brandenburg, B., and Zhuang, X. (2007). Virus trafficking - learning from single-virus tracking. Nat. Rev Microbiol. 5, 197-208.

Brandizzi, F., Snapp, E. L., Roberts, A. G., Lippincott-Schwartz, J., and Hawes, C. (2002). Membrane protein transport between the endoplasmic reticulum and the Golgi in tobacco leaves is energy dependent but cytoskeleton independent: evidence from selective photobleaching. Plant Cell 14, 1293-1309.

Carette, J. E., Stuiver, M., Van Lent, J., Wellink, J., and Van Kammen, A. (2000). Cowpea mosaic virus infection induces a massive proliferation of endoplasmic reticulum but not Golgi membranes and is dependent on de novo membrane synthesis. $J$. Virol. 74, 6556-6563.

Carette, J. E., Van Lent, J., Macfarlane, S. A., Wellink, J., and Van Kammen, A. (2002a). Cowpea mosaic virus 32 - and 60-kilodalton replication proteins target and change the morphology of endoplasmic reticulum membranes. J. Virol. 76, 62936301.

Carette, J. E., Verver, J., Martens, J., Van Kampen, T., Wellink, J., and Van Kammen, A. (2002b). Characterization of plant proteins that interact with cowpea mosaic virus ' $60 \mathrm{~K}$ ' protein in the yeast two-hybrid system. J. Gen. Virol. 83, 885-893.

Carrington, J. C., Jensen, P. E., and Schaad, M. C. (1998). Genetic evidence for an essential role for potyvirus CI protein in cell-to-cell movement. Plant J. 14, 393-400.

Chen, J., Qi, X., and Zheng, H. (2012). Subclass-specific localization and trafficking of Arabidopsis p24 proteins in the ER-Golgi interface. Traffic 13, 400-415.

Chen, J., Stefano, G., Brandizzi, F. and Zheng, H. (2011). Arabidopsis RHD3 mediates the generation of the tubular ER network and is required for Golgi distribution and motility in plant cells. J. Cell Sci. 124, 2241-2252.

Clague, M. J., and Urbe, S. (2001). The interface of receptor trafficking and signalling. J. Cell Sci. 114, 3075-3081.

Cotton, S., Grangeon, R., Thivierge, K., Mathieu, I., Ide, C., Wei, T., et al. (2009). Turnip mosaic virus RNA replication complex vesicles are mobile, align with microfilaments, and are each derived from a single

\section{ACKNOWLEDGMENTS}

This study was supported by the National Science and Engineering Research Council of Canada and from Le Fonds de la Recherche sur la Nature et les Technologies from the Government of Québec to Jean-François Laliberté and Huanquan Zheng.

viral genome. J. Virol. 83, 10460 10471.

Cowan, G. H., Lioliopoulou, F., Ziegler, A., and Torrance, L. (2002). Subcellular localisation, protein interactions, and RNA binding of potato moptop virus triple gene block proteins. Virology 298, 106-115.

daSilva, L. L., Snapp, E. L., Denecke, J., Lippincott-Schwartz, J., Hawes, C. and Brandizzi, F. (2004). Endoplasmic reticulum export sites and Golgi bodies behave as single mobile secretory units in plant cells. Plant Cell 16, 1753-1771.

Dettmer, J., Hong-Hermesdorf, A., Stierhof, Y. D., and Schumacher, K. (2006). Vacuolar H+-ATPase activity is required for endocytic and secretory trafficking in Arabidopsis. Plant Cell 18, 715-730.

de Zoeten, G. A., Assink, A. M. and Van Kammen, A. (1974). Association of cowpea mosaic virusinduced double-stranded RNA with a cytopathological structure in infected cells. Virology 59, 341-355.

Ding, X. S., Liu, J. Z., Cheng, N. H., Folimonov, A., Hou, Y. M., Bao, Y., etal. (2004). The tobacco mosaic virus $126-\mathrm{kDa}$ protein associated with virus replication and movement suppresses RNA silencing. Mol. Plant Microbe Interact. 17, 583-592.

Du, Y., Ferro-Novick, S., and Novick, P. (2004). Dynamics and inheritance of the endoplasmic reticulum. J. Cell Sci. 117, 2871-2878.

Dufresne, P. J., Thivierge, K., Cotton, S., Beauchemin, C., Ide, C., Ubalijoro, E., et al. (2008). Heat shock 70 protein interaction with turnip mosaic virus RNA-dependent RNA polymerase within virus-induced membrane vesicles. Virology 374 , 217-227.

Faso, C., Chen, Y. N., Tamura, K., Held, M., Zemelis, S., Marti, L. etal. (2009). A missense mutation in the Arabidopsis COPII coat protein Sec24A induces the formation of clusters of the endoplasmic reticulum and Golgi apparatus. Plant Cell 21, 3655-3671.

Figueira, A. D., Golem, S., Goregaoker, S. P., and Culvert, J. N. (2002). A nuclear localization signal and a membrane association domain contribute to the cellular localization of the tobacco mosaic virus $126-\mathrm{kDa}$ replicase protein. Virology 301, 81-89.

Genoves, A., Navarro, J. A., and Pallas, V. (2010). The intra- and intercellular movement of melon necrotic spot virus (MNSV) depends on an active secretory pathway. Mol. Plant Microbe Interact. 23, 263-272.

Gorvel, J. P., Chavrier, P., Zerial, M., and Gruenberg, J. (1991). rab5 controls early endosome fusion in vitro. Cell 64, 915-925.

Grangeon, R., Agbeci, M., Chen, J., Grondin, G., Zheng, H., and Laliberte, J. F. (2012). Impact on the endoplasmic reticulum and Golgi apparatus of turnip mosaic virus infection. J. Virol. 86, 9255-9265.

Grant, B. D., and Donaldson, J. G. (2009). Pathways and mechanisms of endocytic recycling. Nat. Rev. Mol. Cell Biol. 10, 597-608.

Gruenberg, J., and Maxfield, F. R. (1995). Membrane transport in the endocytic pathway. Curr. Opin. Cell Biol. 7, 552-563.

Hagiwara, Y., Komoda, K., Yamanaka, T., Tamai, A., Meshi, T., Funada, R., et al. (2003). Subcellular localization of host and viral proteins associated with tobamovirus RNA replication. EMBO J. 22, 344-353.

Hammond, A. T., and Glick, B. S. (2000). Dynamics of transitional endoplasmic reticulum sites in vertebrate cells. Mol. Biol. Cell 11, 3013-3030.

Hanton, S. L., Bortolotti, L. E., Renna, L., Stefano, G., and Brandizzi, F. (2005). Crossing the divide - transport between the endoplasmic reticulum and Golgi apparatus in plants. Traffic 6, 267-277.

Haupt, S., Cowan, G. H., Ziegler, A., Roberts, A. G., Oparka, K. J., and Torrance, L. (2005). Two plantviral movement proteins traffic in the endocytic recycling pathway. Plant Cell 17, 164-181.

Heinlein, M., Padgett, H. S., Gens, J. S., Pickard, B. G., Casper, S. J., Epel, B. L., et al. (1998). Changing patterns of localization of the tobacco mosaic virus movement protein and replicase to the endoplasmic reticulum and microtubules during infection. Plant Cell 10, 1107-1120.

Hirashima, K., and Watanabe, Y. (2001). Tobamovirus replicase coding region is involved in cell-to-cell movement. J. Virol. 75, 8831-8836. 
Hsu, N. Y., Ilnytska, O., Belov, G., Santiana, M., Chen, Y. H., Takvorian, P. M., et al. (2010). Viral reorganization of the secretory pathway generates distinct organelles for RNA replication. Cell 141, 799-811.

Hsu, V. W., and Yang, J. S. (2009). Mechanisms of COPI vesicle formation. FEBS Lett. 583, 3758-3763.

Hu, J., Shibata, Y., Zhu, P. P., Voss, C., Rismanchi, N., Prinz, W. A., et al. (2009). A class of dynamin-like GTPases involved in the generation of the tubular ER network. Cell 138, 549-561.

Huang, Z., Andrianov, V. M., Han, Y., and Howell, S. H. (2001). Identification of Arabidopsis proteins that interact with the cauliflower mosaic virus (CaMV) movement protein. Plant Mol. Biol. 47, 663-675.

Huang, Z., Han, Y., and Howell, S. H. (2000). Formation of surface tubules and fluorescent foci in Arabidopsis thaliana protoplasts expressing a fusion between the green fluorescent protein and the cauliflower mosaic virus movement protein. Virology $271,58-64$.

Hwang, I., and Robinson, D. G. (2009). Transport vesicle formation in plant cells. Curr. Opin. Plant Biol. 12, 660-669.

Kang, B. H., Nielsen, E., Preuss, M. L., Mastronarde, D., and Staehelin, L. A. (2011). Electron tomography of RabA4b- and PI-4Kbetal-labeled trans Golgi network compartments in Arabidopsis. Traffic 12, 313-329.

Katzmann, D. J., Babst, M., and Emr, S. D. (2001). Ubiquitin-dependent sorting into the multivesicular body pathway requires the function of a conserved endosomal protein sorting complex, ESCRT-I. Cell 106, 145-155.

Kikkert, M., Van Lent, J., Storms, M., Bodegom, P., Kormelink, R., and Goldbach, R. (1999). Tomato spotted wilt virus particle morphogenesis in plant cells. J. Virol. 73, 2288-2297.

Kitajima, E. W., Deavila, A. C., Resende, R. D., Goldbach, R. W., and Peters, D. (1992). Comparative cytological and immunogold labeling studies on different isolates of tomato spotted wilt virus. J. Submicrosc. Cytol. Pathol. 24, $1-14$.

Ladinsky, M. S., Mastronarde, D. N., Mcintosh, J. R., Howell, K. E., and Staehelin, L. A. (1999). Golgi structure in three dimensions: functional insights from the normal rat kidney cell. J. Cell Biol. 144, 1135-1149.

Laliberte, J. F., and Sanfacon, H. (2010). Cellular remodeling during plant virus infection. Annu. Rev. Phytopathol. 48, 69-91.
Lee, M. C., and Miller, E. A. (2007). Molecular mechanisms of COPII vesicle formation. Semin. Cell Dev. Biol. 18, 424-434.

Lewandowski, D. J., and Adkins, S. (2005). The tubule-forming NSm protein from tomato spotted wilt virus complements cell-to-cell and long-distance movement of tobacco mosaic virus hybrids. Virology 342 , 26-37.

Lewis, J. D., and Lazarowitz, S. G (2010). Arabidopsis synaptotagmin SYTA regulates endocytosis and virus movement protein cell-to-cell transport. Proc. Natl. Acad. Sci. U.S.A. 107, 2491-2496.

Lucas, W. J. (2006). Plant viral movement proteins: agents for cell-to-cell trafficking of viral genomes. Virology 344, 169-184.

Marti, L., Fornaciari, S., Renna, L., Stefano, G., and Brandizzi, F. (2010). COPII-mediated traffic in plants. Trends Plant Sci. 15, 522-528.

Mas, P., and Beachy, R. N. (1999). Replication of tobacco mosaic virus on endoplasmic reticulum and role of the cytoskeleton and virus movement protein in intracellular distribution of viral RNA. J. Cell Biol. 147, 945-958.

Miller, S., and Krijnse-Locker, J. (2008). Modification of intracellular membrane structures for virus replication. Nat. Rev. Microbiol. 6, 363-374.

Mumford, R. A., Barker, I., and Wood, K. R. (1996). The biology of the tospoviruses. Ann. Appl. Biol. 128, 159-183.

Nebenfuhr, A., Ritzenthaler, C., and Robinson, D. G. (2002). Brefeldin A: deciphering an enigmatic inhibitor of secretion. Plant Physiol. 130, 1102 1108.

Netherton, C., Moffat, K., Brooks, E. and Wileman, T. (2007). A guide to viral inclusions, membrane rearrangements, factories, and viroplasm produced during virus replication. Adv. Virus Res. 70, 101-182.

Nicolas, O., Dunnington, S. W., Gotow L. F., Pirone, T. P., and Hellmann, G. M. (1997). Variations in the VPg protein allow a potyvirus to overcome va gene resistance in tobacco. Virology 237, 452-459.

Nishikiori, M., Mori, M., Dohi, K., Okamura, H., Katoh, E., Naito, S., etal. (2011). A host small GTPbinding protein ARL8 plays crucial roles in Tobamovirus RNA replication. PLoS Pathog 7:e1002409. doi: 10.1371/journal.ppat.1002409

Novoa, R. R., Calderita, G., Arranz, R., Fontana, J., Granzow, H., and Risco, C. (2005). Virus factories: associations of cell organelles for viral replication and morphogenesis. Biol. Cell 97, 147-172.

Orso, G., Pendin, D., Liu, S., Tosetto, J. Moss, T. J., Faust, J. E., et al. (2009). Homotypic fusion of ER membranes requires the dynamin-like GTPase atlastin. Nature 460, 978-983.

Preuss, M. L., Schmitz, A. J., Thole, J. M., Bonner, H. K., Otegui, M. S., and Nielsen, E. (2006). A role for the RabA4b effector protein PI-4Kbetal in polarized expansion of root hair cells in Arabidopsis thaliana. J. Cell Biol. 172, 991-998.

Qi, X., Kaneda, M., Chen, J., Geitmann, A., and Zheng, H. (2011). A specific role for Arabidopsis TRAPPII in postGolgi trafficking that is crucial for cytokinesis and cell polarity. Plant J. 68, 234-248.

Qi, X., and Zheng, H. (2011). Arabidopsis TRAPPII is functionally linked to Rab-A, but not Rab-D in polar protein trafficking in trans-Golgi network. Plant Signal. Behav. 6, 1679 1683.

Reichel, C., and Beachy, R. N. (1998). Tobacco mosaic virus infection induces severe morphological changes of the endoplasmic reticulum. Proc. Natl. Acad. Sci. U.S.A. 95, 11169-11174.

Ribeiro, D., Borst, J. W., Goldbach, R., and Kormelink, R. (2009). Tomato spotted wilt virus nucleocapsid protein interacts with both viral glycoproteins $\mathrm{Gn}$ and $\mathrm{Gc}$ in planta. Virology 383, 121-130.

Ribeiro, D., Foresti, O., Denecke, J., Wellink, J., Goldbach, R., and Kormelink, R. J. (2008). Tomato spotted wilt virus glycoproteins induce the formation of endoplasmic reticulum- and Golgi-derived pleomorphic membrane structures in plant cells. J. Gen. Virol. 89, 18111818.

Rink, J., Ghigo, E., Kalaidzidis, Y., and Zerial, M. (2005). Rab conversion as a mechanism of progression from early to late endosomes. Cell 122, 735-749.

Ritzenthaler, C., Laporte, C., Gaire, F., Dunoyer, P., Schmitt, C., Duval, S., et al. (2002). Grapevine fanleaf virus replication occurs on endoplasmic reticulum-derived membranes. $J$. Virol. 76, 8808-8819.

Rojas, M. R., Zerbini, F. M., Allison, R. F., Gilbertson, R. L., and Lucas, W. J. (1997). Capsid protein and helper component-proteinase function as potyvirus cell-to-cell movement proteins. Virology 237, 283-295.

Schoelz, J. E., Harries, P. A., and Nelson, R. S. (2011). Intracellular transport of plant viruses: finding the door out of the cell. Mol. Plant 4, 813-831.
Sharp, T. M., Guix, S., Katayama, K., Crawford, S. E., and Estes, M. K. (2010). Inhibition of cellular protein secretion by norwalk virus nonstructural protein p22 requires a mimic of an endoplasmic reticulum export signal. PLoS ONE 5:e13130. doi: 10.1371/journal.pone.0013130

Sparkes, I., Tolley, N., Aller, I., Svozil, J., Osterrieder, A., Botchway, S., et al. (2010). Five Arabidopsis reticulon isoforms share endoplasmic reticulum location, topology, and membrane-shaping properties. Plant Cell 22, 1333-1343.

Spitzer, C., Reyes, F. C., Buono, R., Sliwinski, M. K., Haas, T. J., and Otegui, M. S. (2009). The ESCRTrelated CHMP1A and $\mathrm{B}$ proteins mediate multivesicular body sorting of auxin carriers in Arabidopsis and are required for plant development. Plant Cell 21, 749-766.

Storms, M. M., Kormelink, R., Peters, D., Van Lent, J. W., and Goldbach, R. W. (1995). The nonstructural NSm protein of tomato spotted wilt virus induces tubular structures in plant and insect cells. Virology 214, 485-493.

Szumlanski, A. L., and Nielsen, E. (2009). The Rab GTPase RabA4d regulates pollen tube tip growth in Arabidopsis thaliana. Plant Cell 21, 526-544.

Tagami, Y., and Watanabe, Y. (2007). Effects of brefeldin A on the localization of Tobamovirus movement protein and cell-to-cell movement of the virus. Virology 361, 133-140.

Thivierge, K., Cotton, S., Dufresne, P. J., Mathieu, I., Beauchemin, C., Ide, C., et al. (2008). Eukaryotic elongation factor $1 \mathrm{~A}$ interacts with turnip mosaic virus RNA-dependent RNA polymerase and VPg-Pro in virus-induced vesicles. Virology 377 , 216-225.

Thomas, C. L., Bayer, E. M., Ritzenthaler, C., Fernandez-Calvino, L., and Maule, A. J. (2008). Specific targeting of a plasmodesmal protein affecting cell-to-cell communication. PLoS Biol. 6:e7. doi: 10.1371/journal.pbio.0060007

Tilsner, J., Linnik, O., Wright, K. M., Bell, K., Roberts, A. G., Lacomme, C., etal. (2012). The TGB1 movement protein of potato virus $\mathrm{X}$ reorganizes actin and endomembranes into the $\mathrm{X}$-body, a viral replication factory. Plant Physiol. 158, 1359-1370.

Traub, L. M., and Kornfeld, S. (1997). The trans-Golgi network: a late secretory sorting station. Curr. Opin. Cell Biol. 9, 527-533.

Ueda, T., Yamaguchi, M., Uchimiya, H., and Nakano, A. (2001). Ara6, a 
plant-unique novel type Rab GTPase, functions in the endocytic pathway of Arabidopsis thaliana. EMBO J. 20, 4730-4741.

Ueki, S., and Citovsky, V. (2011). To gate, or not to gate: regulatory mechanisms for intercellular protein transport and virus movement in plants. Mol. Plant 4, 782-793.

Verchot, J. (2011). Wrapping membranes around plant virus infection. Curr. Opin. Virol. 1, 388-395.

Voeltz, G. K., Prinz, W. A., Shibata, Y., Rist, J. M., and Rapoport, T. A. (2006). A class of membrane proteins shaping the tubular endoplasmic reticulum. Cell 124, 573-586.

Vogel, F., Hofius, D., and Sonnewald, U. (2007). Intracellular trafficking of potato leafroll virus movement protein in transgenic Arabidopsis. Traffic 8, 1205-1214.

Walter, C. T., and Barr, J. N. (2011). Recent advances in the molecular and cellular biology of bunyaviruses. J. Gen. Virol. 92, 2467-2484.
Wei, T., and Wang, A. (2008). Biogenesis of cytoplasmic membranous vesicles for plant potyvirus replication occurs at endoplasmic reticulum exit sites in a COPI- and COPII-dependent manner. J. Virol. 82, 12252-12264.

Wei, T., Zhang, C., Hong, J. Xiong, R., Kasschau, K. D., Zhou, X., etal. (2010). Formation of complexes at plasmodesmata for potyvirus intercellular movement is mediated by the viral protein $\mathrm{P} 3 \mathrm{~N}$ PIPO. PLoS Pathog 6:e1000962. doi: 10.1371/journal.ppat.1000962

Wright, K. M., Wood, N. T., Roberts, A. G., Chapman, S., Boevink, P. Mackenzie, K. M., et al. (2007). Targeting of TMV movement protein to plasmodesmata requires the actin/ER network: evidence from FRAP. Traffic 8, 21-31.

Yamanaka, T., Ohta, T., Takahashi, M., Meshi, T., Schmidt, R., Dean, C., et al. (2000). TOM1, an Arabidopsis gene required for efficient multiplication of a tobamovirus, encodes a putative transmembrane protein. Proc. Natl. Acad. Sci. U.S.A. 97, 10107-10112.

Yuan, Z. J., Chen, H. Y., Chen, Q., Omura, T., Xie, L. H., Wu, Z. J., et al. (2011). The early secretory pathway and an actin-myosin VIII motility system are required for plasmodesmatal localization of the NSvc4 protein of rice stripe virus. Virus Res. 159, 62-68.

Zheng, H., and Chen, J. (2011). Emerging aspects of ER organization in root hair tip growth: lessons from RHD3 and Atlastin. Plant Signal. Behav. 6, 1710-1713.

Zheng, H., Kunst, L., Hawes, C., and Moore, I. (2004). A GFP-based assay reveals a role for RHD3 in transport between the endoplasmic reticulum and Golgi apparatus. Plant J. 37, 398-414.

Conflict of Interest Statement: The authors declare that the research was conducted in the absence of any commercial or financial relationships that could be construed as a potential conflict of interest.

Received: 03 October 2012; paper pending published: 06 November 2012; accepted: 21 December 2012; published online: 11 January 2013.

Citation: Patarroyo C, Laliberté J-F and Zheng H (2013) Hijack it, change it: how do plant viruses utilize the host secretory pathway for efficient viral replication and spread? Front. Plant Sci. 3:308. doi: 10.3389/fpls.2012.00308

This article was submitted to Frontiers in Plant-Microbe Interaction, a specialty of Frontiers in Plant Science.

Copyright (C) 2013 Patarroyo, Laliberté and Zheng. This is an open-access article distributed under the terms of the Creative Commons Attribution License, which permits use, distribution and reproduction in other forums, provided the original authors and source are credited and subject to any copyright notices concerning any third-party graphics etc. 